\title{
Adenotonsillectomy, Bronchoscopy and Bronchoalveolar Lavage in the Management of Preschool Children with Severe Asthma
}

\author{
Emma Campisi ${ }^{1}$, Myrtha Vargas ${ }^{1}$, May Brydges $^{1}$, Aimee Dubeau $^{2}$, Theo Moraes ${ }^{2}$, Paolo \\ Campisi $^{1}$, and Padmaja Subbarao ${ }^{2}$ \\ ${ }^{1}$ The Hospital for Sick Children \\ ${ }^{2}$ Hospital for Sick Children
}

January 6, 2021

\begin{abstract}
Objective: The potential benefit of a combined adenotonsillectomy and bronchoscopy with bronchoalveolar lavage (TA-B-BAL) in preschool children with asthma has been debated in the literature. We aimed to describe the clinical course of preschool children with severe asthma undergoing this combined procedure. Study Design: This is a retrospective case-control study. Patient Selection: Preschool patients diagnosed with severe asthma who underwent TA-B-BAL treatment between 2012 and 2019 were included as cases. Controls were age and sex matched patients receiving standard asthma care. Methodology: A retrospective patient chart review was conducted. Data on demographics, clinical characteristics, medication use, virology and microbiology from bronchoalveolar lavage, and asthma control questionnaires were collected. Cases and controls were compared with t-tests and regression analysis. Results: Eighteen preschool subjects (mean age $3.19 \pm 1.13$ years) in the case group were matched to eighteen control subjects receiving standard care. A Poisson mixed effects regression analysis revealed reduced risk of oral corticosteroid use (RR $0.39,95 \% \mathrm{CI} 0.18,0.83, \mathrm{p}=0.014)$, reduced emergency department visits (RR $0.36,95 \% \mathrm{CI} 0.17$, $0.75, \mathrm{p}=0.01)$ and reduced risk of asthma exacerbations ( $\mathrm{RR} 0.58,95 \% \mathrm{CI} 0.28,1.20, \mathrm{p}=0.14)$ in cases compared to controls. Ten patients experienced clinically meaningful improvements in TRACK scores after the procedure $(\mathrm{p}<0.001)$. Conclusion: This pilot study provides early evidence that preschool children with severe asthma may benefit from combined adenotonsillectomy and bronchoscopy with bronchoalveolar lavage procedure. The procedure is a useful adjunct for reduction of medication use and hospital visits for preschool age patients with severe asthma.
\end{abstract}

Title: Adenotonsillectomy, Bronchoscopy and Bronchoalveolar Lavage in the Management of Preschool Children with Severe Asthma

Emma S. Campisi, B Kin ${ }^{1}$, Myrtha E. Reyna, $\mathrm{MSc}^{2}$, May Grace Brydges, $\mathrm{MSc}^{2}$, Aimee Dubeau, MSc ${ }^{2}$, Theo J. Moraes, MD, PhD, FRCPC ${ }^{2}$, Paolo Campisi*, MSc, MD, FRCSC ${ }^{1}$, Padmaja Subbarao*, MD, MSc, $\mathrm{FRCPC}^{2,3}$

${ }^{1}$ Department of Otolaryngology - Head \& Neck Surgery, Hospital for Sick Children and University of Toronto, Toronto, Canada

2 Division of Respiratory Medicine, Department of Paediatrics, and Program in Translational Medicine, SickKids Research Institute, The Hospital for Sick Children, Toronto, Canada

${ }^{3}$ Dalla Lana School of Public Health, Division of Epidemiology, University of Toronto, Toronto, Canada

*co-senior authors

Correspondence to:

Padmaja Subbarao MD, MSc 
The Hospital for Sick Children

555 University Avenue

Toronto, Ontario M5G 1X8

Canada

E-mail: padmaja.subbarao@sickkids.ca

Phone Number: 416-813-2196

FAX: 416-813-6246

Keywords: Adenotonsillectomy, asthma, pediatric

Conflict of Interest Statement: The authors do not have any conflict of interest to disclose.

Funding Source: None

Abstract (243/250)

Objective: The potential benefit of a combined adenotonsillectomy and bronchoscopy with bronchoalveolar lavage (TA-B-BAL) in preschool children with asthma has been debated in the literature. We aimed to describe the clinical course of preschool children with severe asthma undergoing this combined procedure.

Study Design : This is a retrospective case-control study.

Patient Selection : Preschool patients diagnosed with severe asthma who underwent TA-B-BAL treatment between 2012 and 2019 were included as cases. Controls were age and sex matched patients receiving standard asthma care.

Methodology : A retrospective patient chart review was conducted. Data on demographics, clinical characteristics, medication use, virology and microbiology from bronchoalveolar lavage, and asthma control questionnaires were collected. Cases and controls were compared with t-tests and regression analysis.

Results: Eighteen preschool subjects (mean age $3.19 \pm 1.13$ years) in the case group were matched to eighteen control subjects receiving standard care. A Poisson mixed effects regression analysis revealed reduced risk of oral corticosteroid use (RR 0.39, 95\% CI 0.18, $0.83, \mathrm{p}=0.014)$, reduced emergency department visits (RR 0.36, $95 \%$ CI $0.17,0.75, \mathrm{p}=0.01$ ) and reduced risk of asthma exacerbations (RR $0.58,95 \%$ CI $0.28,1.20, \mathrm{p}=0.14)$ in cases compared to controls. Ten patients experienced clinically meaningful improvements in TRACK scores after the procedure $(\mathrm{p}<0.001)$.

Conclusion: This pilot study provides early evidence that preschool children with severe asthma may benefit from combined adenotonsillectomy and bronchoscopy with bronchoalveolar lavage procedure. The procedure is a useful adjunct for reduction of medication use and hospital visits for preschool age patients with severe asthma.

\section{Introduction}

Asthma in the preschool period has a variable and unclear prognosis for remission, persistence and progression 1. Rather than a single disease, it is increasingly evident that asthma is a cluster of disorders, related by the presence of common symptoms of wheeze and cough ${ }^{2}$. The heterogeneity in asthma reflects differences in the underlying biology and pathophysiology and therefore response to therapy. Comorbidities such as rhinitis, eczema, obstructive sleep apnea and obesity are associated with poor symptom control and may represent differences in underlying pathophysiology ${ }^{3-6}$. The preschool period is further complicated by a lack of objective measures to diagnose and monitor therapy contributing to sub-optimal asthma control. Consequently, preschool children suffer a disproportionate burden of morbidity in terms of exacerbations and hospitalizations ${ }^{7}$. Several strategies aimed at reducing the burden of co-existing comorbidities show 
some promise in improving asthma control and may point to more precise treatments for preschool asthma $5,8,9$.

Adenotonsillectomy (TA) is one of the most common surgical procedures performed in children to address adenotonsillar hypertrophy, obstructive sleep apnea, and recurrent infection and inflammation ${ }^{10-12}$. Studies exploring the effect of TA in asthmatic populations have revealed numerous benefits for symptom control and quality of life ${ }^{8,9,11,13}$. Studies have noted a decrease in the need for respiratory medications postoperatively $^{8,11,12}$ and an improvement in asthma symptoms ${ }^{5,9}$. Most notably, Saito et al. found $60 \%$ of pediatric patients were able to eliminate all of their medication and $28 \%$ were able to eliminate some of their medications post-operatively ${ }^{9}$. Clinically significant improvements in asthma control test scores have also been reported in the literature, associated with a significant decrease in emergency department visits, oral corticosteroid use ${ }^{5}$, and limitation in activity in children following TA ${ }^{11,13}$. The united airway concept may explain the link between upper airway inflammation and asthma ${ }^{13,14}$. Persistent rhinovirus infection, for example, induces the release of multiple pro-inflammatory cytokines and, in turn, the proliferation of tonsillar lymphocytes ${ }^{14}$. Bacteria in the nasal passage, adenoids, and tonsils can similarly trigger inflammation of the lower airways ${ }^{15}$. Pharyngeal lymphoid tissue is the primary site for proliferation of viruses associated with wheezing ${ }^{14}$. Furthermore, Kaditis, et al. concluded that children with a history of wheezing frequently have more tonsillar hypertrophy than those without wheezing ${ }^{14}$. Bhattacharjee noted an association between airway inflammation, childhood obstructive sleep apnea (OSA) and severe asthma ${ }^{8}$. These findings support the hypothesis that TA may have a role in the management of children with severe asthma ${ }^{16}$.

According to the American Thoracic Society guidelines for infants with recurrent or persistent wheezing, following treatment with bronchodilators, inhaled corticosteroids, or systemic steroids, a flexible fiberoptic bronchosopy and bronchoaleolar lavage is conditionally recommended ${ }^{17}$. A bronchoscopy (B) can provide clinicians with important information regarding airway remodelling and anatomic abnormalities such as airway malacia or compression by vascular structures ${ }^{17-19}$. Furthermore, in 20 case series, 40-60\% of infants with recurrent wheezing had a positive bronchoalveolar lavage (BAL) culture ${ }^{17}$. Patients who identify with lower airway infections, eosinophilia or neutrophilia can have improved symptoms with antibiotic therapy or other treatments ${ }^{17}$. The potential benefits of a combined TA, bronchoscopy and bronchoalveolar lavage (TA-B-BAL) procedure on asthma control has not been previously reported.

The aim of this case-control study was to investigate the potential benefit of performing a combined TA, bronchoscopy and bronchoalveolar lavage procedure in a pediatric cohort of patients with preschool asthma.

Materials and Methods

This is a retrospective case-control study of children attending the asthma clinic at the Hospital for Sick Children, Toronto, Canada. The study was reviewed by the institutional Research Ethics Board at the Hospital for Sick Children (REB\# 1000069481, April 19, 2020). Inclusion criteria for the cases were: 1 . Children younger than 6 years at the baseline visit; 2 . A diagnosis of preschool wheeze by a pediatric respirologist; 3. An assessment by a pediatric otolaryngologist; and 4. Treatment with a combined adenotonsillectomy and flexible bronchoscopy with bronchoalveolar lavage (TA-B-BAL) between 2012 and 2019. Patients were offered the TA-B-BAL procedure if they presented with adenotonsillary hypertrophy or history of recurrent infection. "Preschool asthma" was diagnosed in children by clinicians following the Canadian Thoracic Society guidelines ${ }^{20}$. All children had a history of intermittent symptoms and documented response to inhaled bronchodilators.

All cases were matched one-to-one to a control subject followed in the same asthma clinic. Control subjects were diagnosed with preschool asthma by a pediatric respiratory medicine specialist, were treated with standard medical therapy and had no history of tonsillectomy or adenoidectomy. Matching was based on age at initial visit, sex, and time elapsed between visits. The study was designed to assess the effect of the TA-B-BAL procedure while accounting for the natural progression of asthma with age and standard medical therapy.

Data was retrospectively collected from the hospital charts at the following time points: initial assessment(s) 
in the severe asthma clinic; pre-operative consultation with the otolaryngologist; and at the first and second post-procedure assessments in the severe asthma clinic. Data collected included demographics, clinical characteristics, comorbidies and medication use. Asthma medications were categorized as inhaled corticosteroid monotherapy, leukotriene receptor antagonist (LTRA), long-acting beta2-agonist (LABA) and short-acting beta2-agonist (SABA). A detailed asthma history was also recorded, including the number of asthma exacerbations, oral corticosteroid courses prescribed, emergency department visits, and hospital and intensive care unit (ICU) admissions. Symptoms at baseline and during exacerbations were also collected when available. Finally, cytology and microbiology results from the BAL procedure were recorded (Figure 1) .

\section{Asthma Control Questionnaires}

Asthma control was assessed using age-appropriate standardized questionnaires (Test for Respiratory Asthma Control in Kids(TRACK)) completed by parents with children younger than 5 years. The questionnaire was completed before and after the TA-B-BAL procedure in the case group and at clinic visits for the control group. A higher score indicates greater asthma control and scores greater than 80 indicate that asthma control was achieved ${ }^{21}$.

Information about the number of exacerbations, hospitalizations, emergency room visits, oral steroid courses and pulmonary function tests was collected in a standardized fashion for all patients. A clinically meaningful change in TRACK score was defined as a difference of 10 points ${ }^{22}$. It should be noted that formalized questionnaires were instituted in 2014.

\section{Statistical Analysis}

Baseline demographics, clinical characteristics, and medication use were compared between the TA-B-BAL and control group using unpaired t-tests for continuous variables and Chi-square tests for categorical variables. Differences in the number of exacerbations, oral corticosteroid courses and emergency department visits between the TA-B-BAL and control group were examined by generalized linear mixed effects models (GLMM) with a Poisson distribution function. Changes in TRACK scores between the worst pre-visit and best post-procedure visit were evaluated by a paired t-test within each group.

In all regression models, fixed factors included patient group (TA-B-BAL vs control) and the event (preprocedure vs post-procedure), as well as their interaction. The within-subjects' correlation between preprocedure and post-procedure measures was included by modeling random intercepts by patient.

Statistical analysis was performed using R 3.6.0 ${ }^{23}$ and the "lme4" package ${ }^{24}$.

Results

Between 2012 and 2019, 18 preschool patients (TA-B-BAL) were identified using the inclusion criteria. An additional 18 preschool patients (controls) with severe asthma who attended the asthma clinic during the same time period and did not undergo a TA-B-BAL procedure were identified. The TA-B-BAL and control patients were matched based on age, sex and follow-up time period. The baseline clinical features, comorbidities and demographics of all subjects are summarized inTable $\mathbf{1}$. There were no statistically significant differences between the TA-B-BAL and control group except for anaphylactic food allergy.

The mean (SD) time elapsed between the pre-operative visit and the TA-B-BAL procedure was $3.61 \pm 5.9$ months. Seventeen (94\%) of the cases returned to the asthma clinic for a post-procedure assessment within a mean time of $2.86 \pm 1.7$ months after the TA-B-BAL, and $7.22 \pm 6.06$ after the pre-operative visit. A second follow up assessment occurred for $11(61 \%)$ of the cases $4.3 \pm 4.7$ months after the post-operative visit and $6.9 \pm 4.8$ months after the TA-B-BAL.

The virology and microbiology findings derived from the B and BAL are presented in Figure 2 . Cell counts were also performed revealing neutrophilia in 5 patients $(>15 \%)$ and eosinophilia in 2 patients $(>3 \%)$.

Asthma Exacerbations 
In the year prior to the procedure, patients in the TA-B-BAL group received an average (standard deviation) of 4.17 (4.22) oral steroid courses, attended 5.88 (5.10) emergency department visits, and experienced 5.46 (5.32) asthma wheezing exacerbations. At the post-operative visit, the average number of required oral steroid courses decreased to 1.21 (1.31), the average number of emergency department visits to 1.15 (1.34) and the average number of wheezing exacerbations to 1.77 (1.79).

In the year prior to the first assessment in the asthma clinic, patients in the control group received an average (standard deviation) of 3.47 (2.79) oral steroid courses, attended 3.42 (3.00) emergency department visits, and experienced 3.58 (2.47) asthma wheezing exacerbations. Patients in the control group also demonstrated an improvement with standard asthma treatment. At a time interval matched with the post-operative visit, the mean number of oral corticosteroid courses was 1.89 (2.40), the mean number of emergency department visits was 2.11 (2.85), and the mean number of exacerbations was 1.71 (1.59).

A Poisson mixed effects regression analysis revealed that TA-B-BAL was associated with a $61 \%$ reduced risk of oral corticosteroid use (RR 0.39, 95\% CI 0.18, 0.83, $\mathrm{p}=0.014$ ), $64 \%$ reduction in emergency department visits (RR $0.36,95 \%$ CI $0.17,0.75, \mathrm{p}=0.007)$ and $42 \%$ reduction in the risk of exacerbations (RR $0.58,95 \%$ CI $0.28,1.20, \mathrm{p}=0.14$ ) compared to standard asthma care.

\section{Medication Use}

Trends in asthma medication use by patients in the TA-B-BAL and control group are illustrated in Figure 3 . Four patients from the TA-B-BAL group were able to discontinue all respiratory medications at the time of the second post-operative visit. The average inhaled corticosteroid (ICS) dose at initial assessment was higher in the TA-B-BAL group $(250.0 \mathrm{mg})$ compared to the controls $(100.0 \mathrm{mg})$. Following medical management, the average ICS dose in the TA-B-BAL group was $193.8 \mathrm{mg}$. Post operatively, ICS dose in the TA-B-BAL group decreased to $150.0 \mathrm{mg}$. At a time point matched with the TA-B-BAL group post-operative visit, the controls average ICS dose was $210.4 \mathrm{mg}$ following standard medical management of asthma (Figure 4 ).

\section{Asthma Control Questionnaires}

Longitudinal TRACK scores were available for 10 TA-B-BAL patients. The analysis included questionnaires within a 2-year period before and after the combined procedure. A paired t-test revealed a clinically significant improvement in TRACK score ( $>10$ points change) between the pre-procedure and post-procedure visit $(\mathrm{p}=<0.001)$ in all 10 subjects, with a mean difference of 40.0 points. Four patients $(40 \%)$ reported TRACK scores of $>80$ indicating asthma control at the post-procedure visit.

Longitudinal TRACK scores were available for 12 patients in the control group. Eight patients (66\% of the control group) achieved a clinically significant improvement in TRACK score between the baseline and follow up visit, the overall group mean difference of 25.4 points was significant $(\mathrm{p}=0.001)$. Five patients $(42 \%)$ reported TRACK scores of $>80$ indicating asthma control at the follow-up time point (Figure 5 ).

\section{Discussion}

This case-control study evaluated the effect of a TA-B-BAL procedure in a cohort of preschool children with asthma. This is the first study to report the beneficial effect of a combined TA-B-BAL procedure in a preschool age sample using multiple outcome measures. The available literature of TA in asthma is limited to children with mean age greater than 6 years, studies that examine the impact of adenotonsillectomy only, or the use of less robust asthma control measures.

There are several studies that have examined the impact of adenotonsillectomy only for asthma management. Adenotonsillectomy has been shown to improve asthma symptoms ${ }^{9,13}$ and asthma control questionnaire scores $(\mathrm{ACT} \text { and } \mathrm{CACT})^{11,13,25,26}$, reduce the rate of exacerbations and emergency department visits $5,11,13,25,26$, and decrease the requirement for asthma medication for maintenance and acute exacerbations ${ }^{8,9,11-13,25,26}$. In the present study, patients in the TA-B-BAL group were referred to the clinic on higher doses of ICS compared to the asthmatic controls. Despite these higher doses of ICS, there was little improvement in symptoms noted at the medical follow-up visit, leading to a referral to the otolaryngology service for the 
combined procedure (TA-B-BAL). This contrasts to the asthma control group who achieved greater symptom control at the follow-up visit and at the same ICS dose. Notably, post TA-B-BAL, patienyd in this group were able to achieve the same level of control as those medically managed but at a lower ICS dose.

The vast majority of the studies exploring adenotonsillectomy in pediatric asthma patients included children with a mean age greater than 6 years $5,8,11,13,25,26$. The mean age of the cohort of patients in the present study was notably lower at $3.19 \pm 1.13$ years. Interestingly, Koenigs and colleagues noted that younger children were more likely to demonstrate a post-surgical improvement in asthma control ${ }^{25}$. This was attributed to a number of factors including a higher relative degree of airway obstruction and susceptibility to viral infection in the younger age groups ${ }^{27}$.

Asthma is a heterogenous syndrome, particularly in preschool children. This study and others show that management of upper airway conditions can improve asthma symptom control in a select group of patients ${ }^{5,8,9}$. From a physiological perspective, this may be explained by the united airway hypothesis ${ }^{16}$. This hypothesis suggests that the inflammatory processes from infection in the lower airways may lead to inflammation and proliferation of the lymphadenoid tissues and tonsillar lymphocytes in the upper airway andvice versa 14,16 . Levin and colleagues noted an improvement in markers of airway inflammation (decrease in circulating chitinase activity) in asthmatics who underwent adenotonsillectomy ${ }^{13}$. Alternatively, in preschool children, the pathophysiologic cause of the symptoms may be due to the upper respiratory obstruction mimicking symptoms of asthma. Further studies are needed in this age group to objectively measure airway hyperresponsiveness to further elucidate the underlying pathophysiology.

Asthmatic patients are at an increased risk of respiratory complications following a general anesthetic and surgery ${ }^{28}$. Formal pulmonary evaluation and optimization prior to surgery should be achieved in all asthmatic patients ${ }^{29}$. Nonetheless, airway interventions can be performed safely in preschool wheeze. In a series of 93 children, Busino and colleagues reported no significant difference in the length of hospital stay between asthmatic and non-asthmatic children undergoing adenotonsillectomy ${ }^{11}$. The safety of bronchoscopy and BAL procedures in patients with asthma has also been evaluated and is associated with a very low incidence of major complications $(<2 \%)^{30-32}$. In the present study, only 1 patient experienced respiratory difficulty and pneumonia requiring admission post-operatively. This patient fully recovered without further complication.

BAL procedures are important for the identification of viral and bacterial colonization or infection and for cytology to identify an increase in cells associated with inflammation ${ }^{30,33}$. In line with conditional recommendations put forth by the American Thoracic Society, B and BAL can minimize unecessary antibiotic therapy and ineffective medications by providing accurate culture and cytology data, and identification of anatomical abnormalities ${ }^{17}$. Viral infections cause approximately $85 \%$ of severe asthma exacerbations in children by increasing the production of pro-inflammatory cytokines and chemokines, leading to airway inflammation ${ }^{6,31}$. Furthermore, colonization of the hypopharyngeal region with Streptococcus pneumonia ,Haemophilus influenza and Moraxella catarrhalis has been associated with acute severe exacerbations and hospitalizations for wheeze ${ }^{6}$. Eller and colleagues reported that patients with controlled severe asthma had high eosinophil counts in sputum compared to patients with severe, therapy-resistant asthma ${ }^{34}$. Patients with severe asthma resistant to corticosteroid treatment also have increased neutrophil counts in peripheral blood and BAL specimens ${ }^{34,35}$. In the present study, Moraxella catarrhalis was identified in 11 patients, Streptococcus pneumoniae was identified in 4 patients, and enterovirus/rhinovirus species were isolated in 4 patients. Five patients exhibited neutrophilia $(>15 \%)$ and 2 patients exhibited eosinophilia $(>3 \%)$.

This study has demonstrated that a combined TA-B-BAL procedure can be performed in a cohort of preschool age patients with severe asthma or wheeze. The intervention alleviated symptoms of upper airway obstruction, identified microbiological and cytological characteristics of the lower airways, and ultimately improved measures of asthma control.

\section{Limitations}

Patients with preschool wheeze were offered the combined TA-B-BAL procedure only if they presented with severe wheeze and adenotonsillary hypertrophy or a history of recurrent tonsillar infection. The strict 
inclusion criteria and the concern of operative risk resulted in a small sample size. The small sample size was inadequate to appreciate differences in ICU and hospital admissions. These are rare events and a larger study sample is needed to evaluate this potential complication in preschool children with asthma. In addition, a longer follow up period would be ideal to monitor long term changes in asthma outcomes such as medication use and symptom control.

There are inherent limitations of a retrospective design such as lack of randomization of subjects and incomplete or missing data. The latter was evident with the TRACK questionnaires, as this tool was not implemented prior to 2014 . As such, only $56 \%$ of patients completed the questionnaires both pre and postprocedure or treatment.

Conclusion

This exploratory study has provided subjective and objective evidence in support of a combined TA-B-BAL procedure to improve asthma control in preschool aged patients. Patients with a positive BAL were able to receive appropriate therapies to improve their symptoms. The combined TA-B-BAL procedure significantly reduced oral corticosteroid use and emergency department visits and lead to clinically meaningful improvements in TRACK scores. Although this is not a large study, there was sufficient experience to draw positive conclusions. Randomized control trials and prospective studies with larger sample size and longer follow up are required to better define the benefits of a TA-B-BAL procedure.

Acknowledgements: None to disclose

References

1. To T, Gershon A, Wang C, Dell S, Cicutto L. Persistence and Remission in Childhood Asthma: A Population-Based Asthma Birth Cohort Study.Arch Pediatr Adolesc Med. 2007;161(12):1197-1204.

2. Pavord ID, Beasley R, Agusti A, et al. After asthma: redefining airways diseases. Lancet. 2018;391(10118):350-400.

3. Bousquet J, Jeffery PK, Busse WW, Johnson M, Vignola AM. Asthma.Am J Respir Crit Care Med. 2000;161(5):1720-1745.

4. de Groot EP, Nijkamp A, Duiverman EJ, Brand PLP. Allergic rhinitis is associated with poor asthma control in children with asthma. Thorax. 2012;67(7):582.

5. Kheirandish-Gozal L, Dayyat EA, Eid NS, Morton RL, Gozal D. Obstructive sleep apnea in poorly controlled asthmatic children: Effect of adenotonsillectomy. Pediatr Pulmonol. 2011;46(9):913-918.

6. Ramratnam SK, Visness CM, Jaffee KF, et al. Relationships among maternal stress and depression, type 2 responses, and recurrent wheezing at age 3 years in low-income urban families. Am J Respir Crit Care Med. 2017;195(5):674-681.

7. Chen W, Subbarao P, McGihon RE, et al. Patterns of health care use related to respiratory conditions in early life: A birth cohort study with linked administrative data. Pediatr Pulmonol.2019;54(8):1267-1276.

8. Bhattacharjee R, Choi BH, Gozal D, Mokhlesi B. Association of Adenotonsillectomy with Asthma Outcomes in Children: A Longitudinal Database Analysis. PLOS Med. 2014;11(11):e1001753.

9. Saito H, Asakura K, Hata M, Kataura A, Morimoto K. Does adenotonsillectomy affect the course of bronchial asthma and nasal allergy? ACTA Otolaryngol. 1996;523:212-215.

10. Baum ED. Tonsillectomy and adenoidectomy and myringotomy with tube insertion. Pediatr Rev. 2010;31(10):417-425.

11. Busino RS, Quraishi HA, Aguila HA, Montalvo E, Connelly P. The impact of adenotonsillectomy on asthma in children. Laryngoscope.2010;120:S221-S221. 
12. Piessens P, Hens G, Lemkens N, Schrooten W, Debruyne F, Lemkens P. Effect of adenotonsillectomy on the use of respiratory medication.Int J Pediatr Otorhinolaryngol. 2012;76(6):906-910.

13. Levin JC, Gagnon L, He X, Baum ED, Karas DE, Chupp GL. Improvement in asthma control and inflammation in children undergoing adenotonsillectomy. Int J Pediatr Res. 2013;75:403.

14. Kaditis AG, Kalampouka E, Hatzinikolaou S, et al. Associations of tonsillar hypertrophy and snoring with history of wheezing in childhood.Pediatr Pulmonol. 2010;45(3):275-280.

15. Lai L, Hopp RJ, Lusk RP. Pediatric Chronic Sinusitis and Asthma: A Review. J Asthma. 2006;43(10):719725.

16. Gozal D. Pediatric OSA: A case for "United we stand" in the way of a breath. Pediatr Pulmonol. 2010;45(12):1151-1152.

17. Boyer D, Barsky E, Papantonakis CM, et al. Diagnostic Evaluation of Infants with Recurrent or Persistent Wheezing. Ann Am Thorac Soc.2016;13(11):2057-2059.

18. Januska MN, Goldman DL, Webley W, et al. Bronchoscopy in severe childhood asthma: Irresponsible or irreplaceable? Pediatr Pulmonol. 2020;55(3):795-802.

19. Mazurek P, Kukiełka K, Pajdowska M, Wojnicka J, Markiewicz B. Experimental methods of treatment used during the bronchoscopy.EJMT. 2020;2:27.

20. Ducharme FM, Dell SD, Radhakrishnan D, et al. Diagnosis and management of asthma in preschoolers: A Canadian Thoracic Society and Canadian Paediatric Society position paper. Paediatr Child Health. 2015;20(7):353-361.

21. Dinakar C, Chipps BE. Clinical Tools to Assess Asthma Control in Children. Pediatrics. 2017;139(1):e20163438.

22. Zeiger RS, Mellon M, Chipps B, et al. Test for Respiratory and Asthma Control in Kids (TRACK): Clinically meaningful changes in score.J Allergy Clin Immunol. 2011;128(5):983-988.

23. R: A language and environment for statistical computing[computer program]. Vienna, Austria2019.

24. Douglas Bates MM, Ben Bolker, Steve Walker. Fitting Linear Mixed-Effects Models Using lme4. J Statl Softw. 2015;67(1):1-48.

25. Koenigs M. Predictors And Potential Mechanisms Of Improvement In Asthma Control In Children Following Adenotonsillectomy. 2014.

26. Goldstein NA, Thomas MS, Yu Y, et al. The impact of adenotonsillectomy on pediatric asthma. Pediatr Pulmonol.2019;54(1):20-26.

27. Fasano MB. Combined airways: impact of upper airway on lower airway. Curr Opin Otolaryngol Head Neck Surg. 2010;18(1):15-20.

28. Kalra M, Buncher R, Amin RS. Asthma as a risk factor for respiratory complications after adenotonsillectomy in children with obstructive breathing during sleep. Ann Allergy, Asthma Immunol.2005;94(5):549-552.

29. Orestes MI, Lander L, Verghese S, Shah RK. Incidence of laryngospasm and bronchospasm in pediatric adenotonsillectomy. Laryngoscope.2012;122(2):425-428.

30. Saglani S, Payne DNR, Nicholson AG, Scallan M, Haxby E, Bush A. The safety and quality of endobronchial biopsy in children under five years old. Thorax. 2003;58(12):1053.

31. Ciepiela O, Ostafin M, Demkow U. Neutrophils in asthma-A review.Respir Physiol Neurobiol. 2015;209:13-16. 
32. Humbert M, Robinson DS, Assoufi B, Kay AB, Durham SR. Safety of fibreoptic bronchoscopy in asthmatic and control subjects and effect on asthma control over two weeks. Thorax. 1996;51(7):664.

33. de Blic J, Marchac V, Scheinmann P. Complications of flexible bronchoscopy in children: prospective study of 1,328 procedures.Eur Respir J. 2002;20(5):1271.

34. Eller MCN, Vergani KP, Saraiva-Romanholo BM, Antonangelo L, Leone C, Rodrigues JC. Can inflammatory markers in induced sputum be used to detect phenotypes and endotypes of pediatric severe therapy-resistant asthma? Pediatr Pulmonol. 2018;53(9):1208-1217.

35. Cowburn AS, Condliffe AM, Farahi N, Summers C, Chilvers ER. Advances in Neutrophil Biology: Clinical Implications. Chest.2008;134(3):606-612.
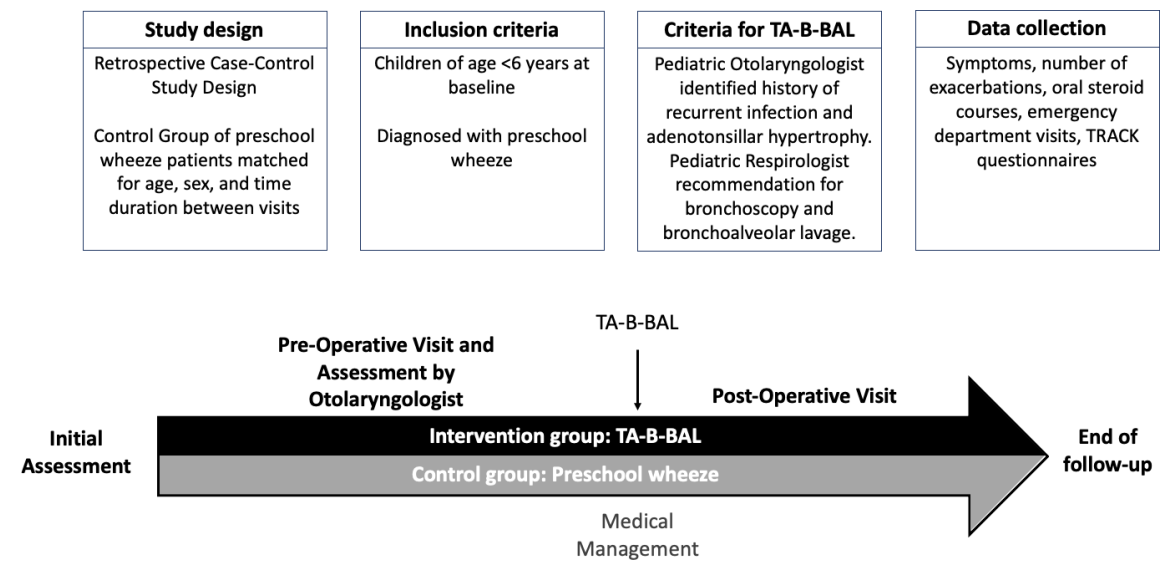
Bronchoalveolar Lavage- Bacteria

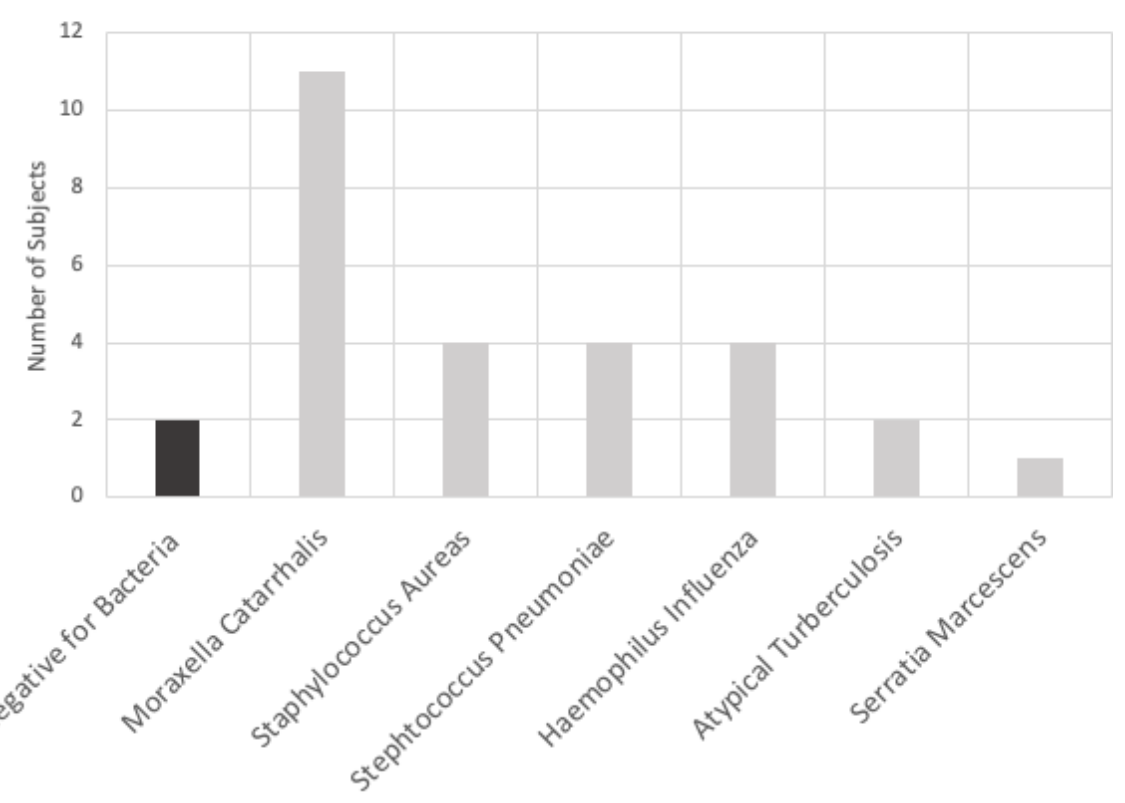

Bronchoalveolar Lavage- Viruses

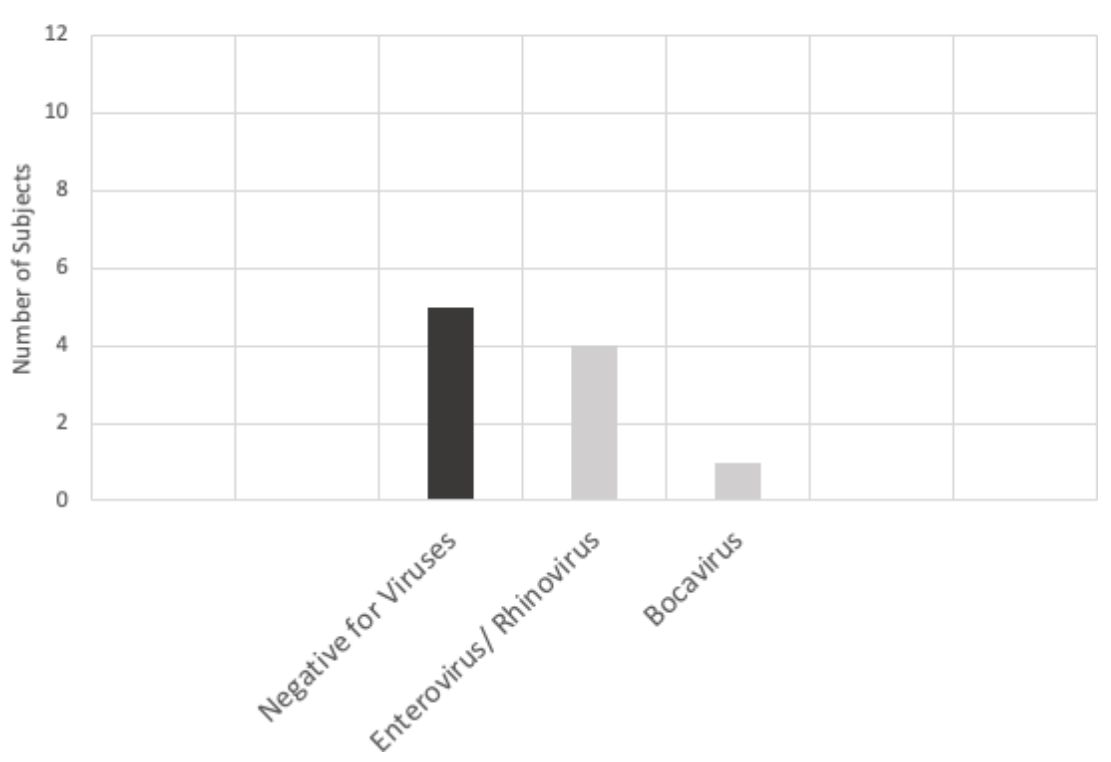



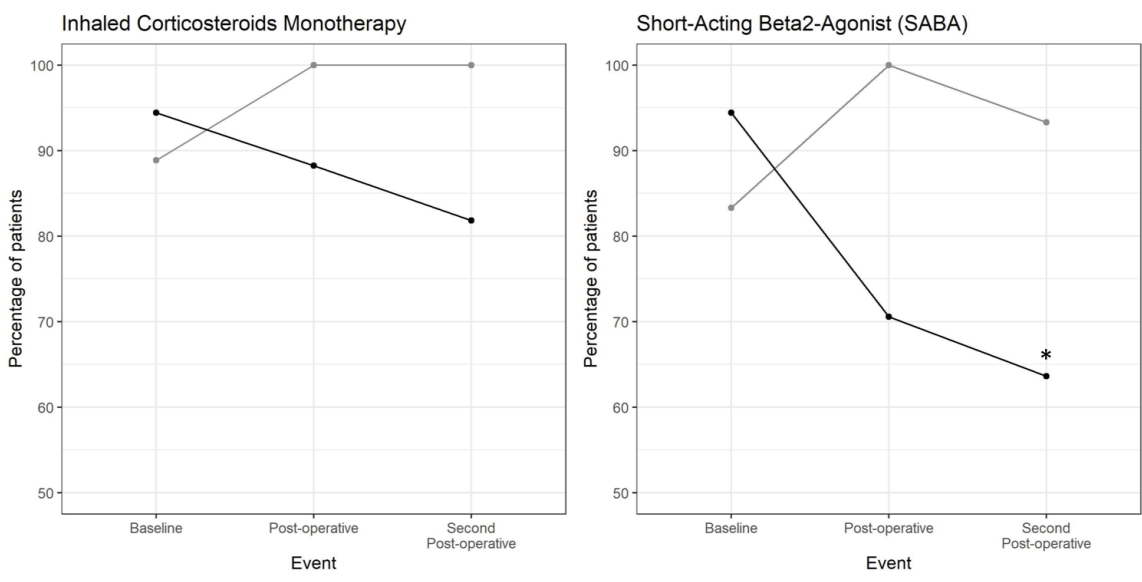

$\rightarrow$ Asthma Control $\rightarrow$ TA-B-BAL

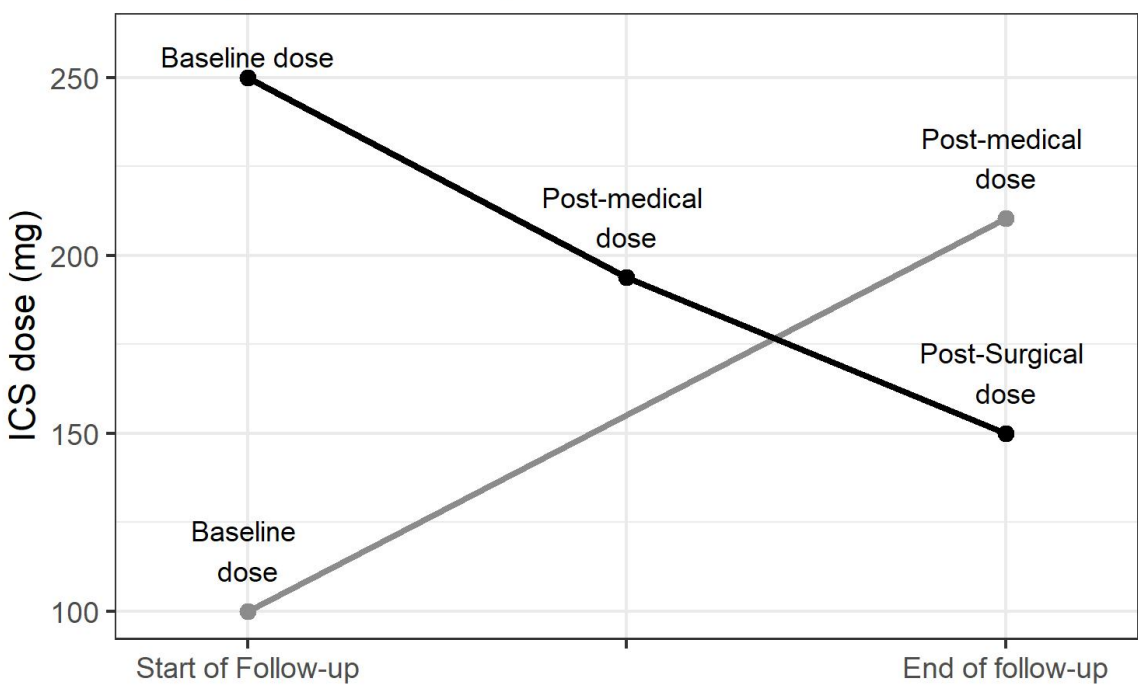

$\because$ Asthma Control $\multimap$ TA-B-BAL 


\section{Relationship between TRACK score by intervention group}

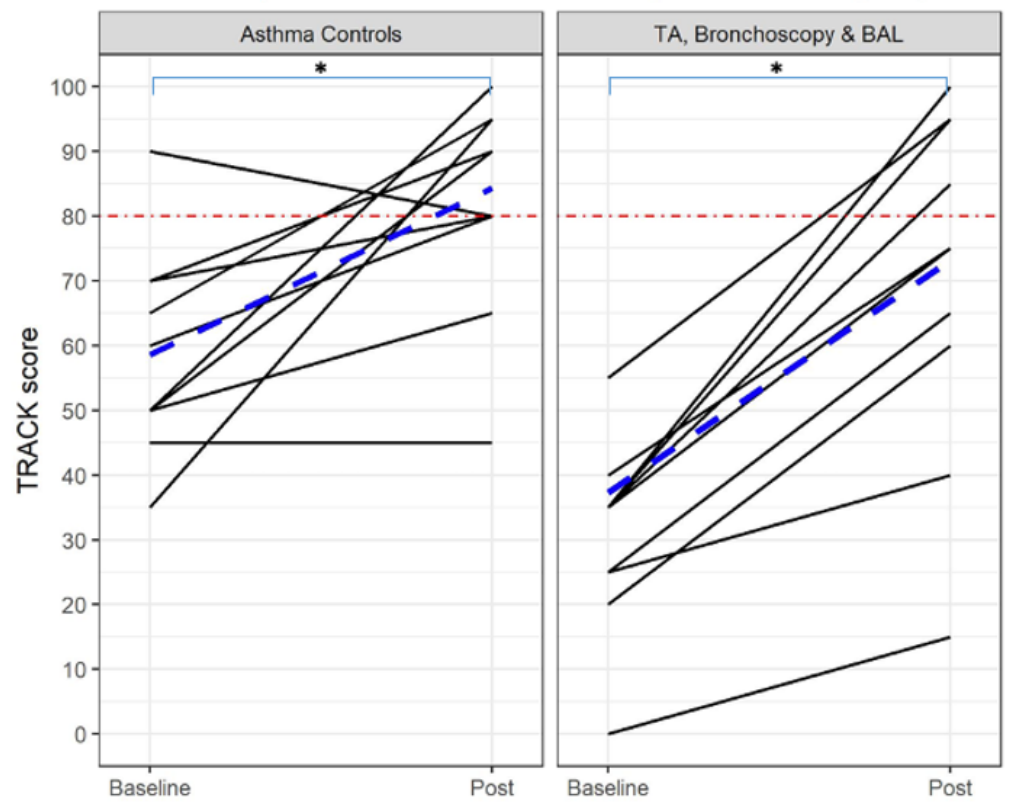

\section{Hosted file}

Table 1_Campisi.pdf available at https://authorea.com/users/387818/articles/502742adenotonsillectomy-bronchoscopy-and-bronchoalveolar-lavage-in-the-management-ofpreschool-children-with-severe-asthma 